\title{
Some basic features of the beam emittance
}

\author{
Klaus Floettmann* \\ DESY, Notkestrasse 85, 22603 Hamburg, Germany \\ (Received 14 November 2002; published 6 March 2003)
}

\begin{abstract}
Some general features of the beam emittance are discussed. The significance of rms values for the emittance calculation and emittance measurements is emphasized and it is shown that the emittance of a beam is not necessarily constant in a drift space.
\end{abstract}

DOI: 10.1103/PhysRevSTAB.6.034202

PACS numbers: 29.27.Fh

\section{INTRODUCTION}

The beam emittance as a conserved quantity of motion evolves out of the Hamilton formalism and is hence based on canonical coordinates. In case that no transverse vector potential exists in the region of the beam path, e.g., no solenoid fields act on the beam, the transverse canonical coordinates are the transverse particle positions $x$ and $y$ and the transverse mechanical momenta $p x$ and $p y$. According to Liouville's theorem the six dimensional phase space density along any particle trajectory is a conserved quantity. The emittance is a six dimensional phase space volume which is enclosed by a five dimensional surface of constant density. In order to relate the six dimensional emittance to quantities which are meaningful to accelerator physics or physicists at least three steps have to be taken:

1. Instead of a six dimensional phase space volume the areas of the projections onto three orthogonal planes, i.e., $x$ - $p x, y-p y$, and $z-p z$, are considered.

2 . The emittance calculation is in general restricted to the high density core of the distribution, rather than to the complete area. This is achieved by an appropriate scaling parameter (e.g., the rms value) which allows one, in case of known distributions, to calculate the fraction of particles inside the considered area.

3. Instead of the phase space area in $x$-px and $y$-py the identities $x^{\prime}=p_{x} / p_{z}$ and $y^{\prime}=p_{y} / p_{z}$ are used and the socalled trace-space areas in the $x-x^{\prime}$ and $y-y^{\prime}$ planes are considered.

In the following the calculation of an appropriate scaling parameter will be discussed first. It will be shown how the beam emittance calculated in trace space is related to the emittance in phase space before some peculiarities of the emittance are described, which originate in the projection of the phase space volume onto the orthogonal phase space planes. Finally the effect of the longitudinal coordinate onto the calculated transverse emittance will be discussed. Only $x, p x$, and $x^{\prime}$ will be mentioned; all formulas apply equivalently to $y$, $p y$, and $y^{\prime}$.

*Electronic address: Klaus.Floettmann@DESY.De

\section{SECOND MOMENT OF ARBITRARY PARTICLE DISTRIBUTIONS}

The second central moment or mean square value of a two dimensional distribution $\rho(x, y)$ is given by

$$
x_{\mathrm{rms}}^{2}=\frac{\int x^{2} \rho(x, y) d x d y}{\int \rho(x, y) d x d y}-\left(\frac{\int x \rho(x, y) d x d y}{\int \rho(x, y) d x d y}\right)^{2} .
$$

The denominator normalizes the result and the second term in Eq. (1) shifts the distribution to its mean position so that the root mean square (rms) value is independent of the average position and the height but is related only to the form of the distribution. The interesting part is the integral of the form $\int x^{2} \rho(x, y) d x d y$. For the emittance calculation it is important to know how the rms value of a two dimensional distribution develops if the distribution is rotated by an angle $\varphi$ around the origin of the coordinate system. This is included in the integral as

$$
\int x^{2} \rho(x \cdot \cos \varphi-y \cdot \sin \varphi, x \cdot \sin \varphi+y \cdot \cos \varphi) d x d y .
$$

Introducing the new variables:

$$
x_{0}=x \cdot \cos \varphi-y \cdot \sin \varphi, y_{0}=x \cdot \sin \varphi+y \cdot \cos \varphi,
$$

$x$ rewrites as

$$
x=x_{0} \cdot \cos \varphi+y_{0} \cdot \sin \varphi,
$$

and the integral can be written in the form:

$$
\int\left(x_{0} \cdot \cos \varphi+y_{0} \cdot \sin \varphi\right)^{2} \rho\left(x_{0}, y_{0}\right) d x_{0} d y_{0}
$$

The mixed term in the square sum cancels in the integral, thus the second central moment or mean square value is -independent of all the details of the distribution - of the form:

$$
\begin{aligned}
x_{\mathrm{rms}}^{2} & =\sigma x_{0}^{2} \cdot \cos ^{2} \varphi+\sigma y_{0}^{2} \cdot \sin ^{2} \varphi \\
& =\sigma x_{0}^{2}+\left(\sigma y_{0}^{2}-\sigma x_{0}^{2}\right) \sin ^{2} \varphi,
\end{aligned}
$$

where $\sigma x_{0}^{2}$ and $\sigma y_{0}^{2}$ are the maximum and minimum mean square values of the distribution.

This is a remarkable result. Since Eq. (6) is valid for all distributions we know without further calculations that 
the rms values of any rotating distribution are described by the rotation of an ellipse, because the rms values of a double Gaussian distribution are known to describe an ellipse. For a formal, mathematical proof, that Eq. (6) describes an ellipse, one may start from a parametric representation of an ellipse rotated by an angle $\varphi$ :

$$
\begin{aligned}
& x=x_{0} \cos \varphi \cos \theta-y_{0} \sin \varphi \sin \theta, \\
& y=x_{0} \sin \varphi \cos \theta+y_{0} \cos \varphi \sin \theta, \\
& \theta=\left[\begin{array}{ll}
0,2 \pi] .
\end{array}\right.
\end{aligned}
$$

The maximum extension in $x$ is found from $\frac{d x}{d \theta}=0$ at an angle $\theta=\theta_{\max }$ given by

$$
\tan \theta_{\max }=-\frac{y_{0}}{x_{0}} \tan \varphi .
$$

Using Eq. (8) to replace $\theta$ in the first of Eq. (7) yields after some algebraic manipulations Eq. (6).

The ratio $\frac{y}{x}$ at $\theta=\theta_{\max }$ yields the angle of the correlation axis $\varphi_{\text {cor }}$ (see Fig. 1):

$$
\tan \varphi_{\text {cor }}=-\frac{1}{2} \tan 2 \varphi \frac{y_{\max }^{2}-x_{\max }^{2}}{x_{\max }^{2}}
$$

$y_{\max }$ is defined equivalent to $x_{\max }$ as the maximum extension in $y$. The axis defined by the angle $\varphi_{\text {cor }}$ is not a principle axis of the ellipse, but it is a symmetry axis in the sense that at any position $x$ the distance $\Delta y$ between the axis and the upper and the lower branches of the ellipse is equal as indicated in Fig. 1. Thus the line defined by $\varphi_{\text {cor }}$ corresponds to a line obtained by a straight line fit through the distribution with an rms minimization. While the angle $\varphi$ defines the rotation angle of the principle axes of the ellipse, $\varphi_{\text {cor }}$ is related to a shearing of the ellipse. If the ellipse is sheared by an angle $\varphi_{\text {cor }}$ the principle axes of the ellipse will be on the axes of the coordinate system as in the case of a rotation by an angle $\varphi$.

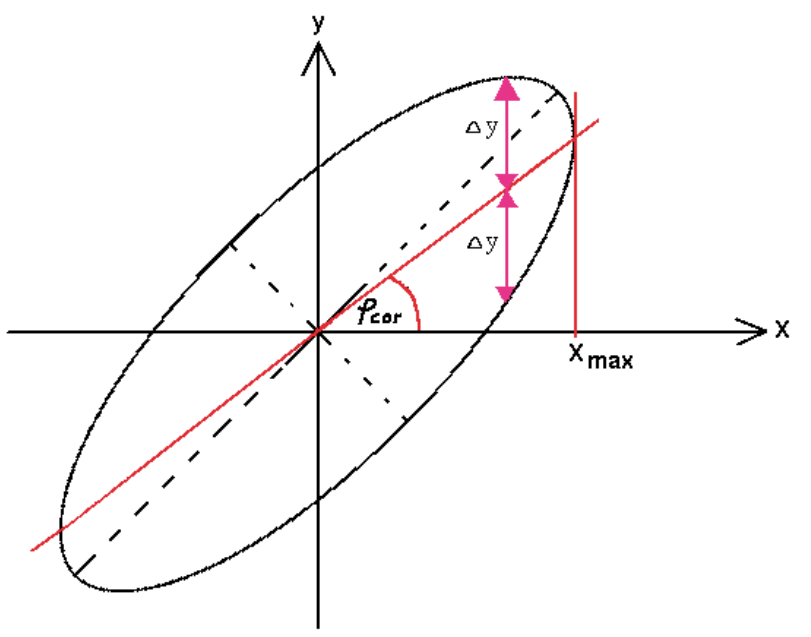

FIG. 1. (Color) Ellipse with principle axes and correlation axis.
Looking back to Eq. (6) and its derivation one may ask, whether the rms value of a distribution is the only scaling parameter which describes an ellipse when the distribution is rotated. The full width at half maximum (FWHM), for example, is often used in experiments, since it is insensitive to long tails and easier determined than the rms value. Writing the FWHM as

$$
\mathrm{FWHM}=f(\varphi) \cdot x_{\mathrm{rms}},
$$

it is obvious that only in the case of a regular distribution where $f(\varphi)$ is constant the FWHM or any other scaling parameter describes an ellipse. Fundamental accelerator physics concepts like the standard optical functions $\alpha, \beta$, and $\gamma$, or the envelope equation are related to the description of an ellipse in phase space. These concepts are the basis of measurements, e.g., of the beam emittance and can hence in the case of irregular distributions be applied only to the rms values (or multiples of the rms values) of the measured distributions.

It should also be noted that an equivalent derivation of Eq. (6) can be made for higher order moments of the distribution, which will end up with different geometrical figures in phase space, so that an optics for higher order moments of distributions is conceivable.

\section{DEFINITION OF THE RMS EMITTANCE}

Based on the previous discussion a statistical definition of the emittance, which is appropriate, for example, for the emittance calculation in computer codes, is the socalled normalized rms emittance defined as

$$
\varepsilon_{n, \mathrm{rms}}=\frac{1}{m_{0} c} \sqrt{\left\langle x^{2}\right\rangle\left\langle p_{x}^{2}\right\rangle-\left\langle x p_{x}\right\rangle^{2}}
$$

where \langle\rangle defines the second central moment of the particle distribution:

$$
\begin{aligned}
\left\langle x^{2}\right\rangle & =\frac{\sum x^{2}}{n}-\left(\frac{\sum x}{n}\right)^{2}, \quad\left\langle p_{x}^{2}\right\rangle=\frac{\sum p_{x}^{2}}{n}-\left(\frac{\sum p_{x}}{n}\right)^{2}, \\
\left\langle x p_{x}\right\rangle & =\frac{\sum x p_{x}}{n}-\frac{\sum x \sum p_{x}}{n^{2}},
\end{aligned}
$$

and all sums are performed for the $n$ particles in the distribution, i.e., $\sum x=\sum_{i=1}^{n} x_{i}$.

$\varepsilon_{n, \text { rms }}$ is proportional to the area of the phase space ellipse; a constant factor $\pi$ is neglected. From the normalized emittance the beam emittance, also called geometric emittance, is derived as

$$
\varepsilon_{\mathrm{rms}}=\frac{1}{\bar{p}_{z}} \varepsilon_{n, \mathrm{rms}},
$$

where $\bar{p}_{z}$ denotes the average longitudinal momentum. Equation (13) displays the well-known adiabatic damping of the emittance with the average longitudinal beam momentum. 
The trace-space emittance $\varepsilon_{\mathrm{tr}, \text { rms }}$ is defined as

$$
\varepsilon_{\mathrm{tr}, \mathrm{rms}}=\sqrt{\left\langle x^{2}\right\rangle\left\langle x^{\prime 2}\right\rangle-\left\langle x x^{\prime}\right\rangle^{2}},
$$

and, to be complete, a normalized trace-space emittance $\varepsilon_{n, \text { tr, rms }}$ can be defined as

$$
\varepsilon_{n, \mathrm{tr}, \mathrm{rms}}=\frac{\bar{p}_{z}}{m_{0} c} \sqrt{\left\langle x^{2}\right\rangle\left\langle x^{\prime 2}\right\rangle-\left\langle x x^{\prime}\right\rangle^{2}} .
$$

$\left\langle x^{\prime 2}\right\rangle$ can be written as

$$
\left\langle x^{\prime 2}\right\rangle=\frac{1}{n} \sum \frac{p_{x}^{2}}{p_{z}^{2}}=\frac{1}{n^{2}} \sum p_{x}^{2} \sum \frac{1}{p_{z}^{2}}
$$

where for simplicity it is assumed that the mean $x^{\prime}$ is zero and that $p x$ and $p z$ are statistically independent. Writing $p_{z}=\bar{p}_{z}+\Delta p$ the sum over $1 / p_{z}^{2}$ can be expanded as

$$
\frac{1}{n} \sum \frac{1}{p_{z}^{2}} \cong \frac{1}{n} \sum \frac{1}{\bar{p}_{z}^{2}}\left[1+\frac{3}{8}\left(\frac{\Delta p}{\bar{p}_{z}}\right)^{2}\right] \cong \frac{1}{\bar{p}_{z}^{2}} .
$$

A linear term in $\Delta p / \bar{p}_{z}$ has already been dropped, since the sum yields zero. Thus $\varepsilon_{\text {tr rms }}=\varepsilon_{\text {rms }}$ in case that the beam is not divergent or convergent, i.e., close to a waist where $\left\langle x x^{\prime}\right\rangle \cong 0$. In case of a correlated transverse momentum spread $x^{\prime}$ can be written as

$$
p_{x}=p_{x, \text { inc }}+C \cdot x, \quad x^{\prime}=\frac{p_{x, \text { inc }}}{p_{z}}+\frac{C \cdot x}{p_{z}} .
$$

$C$ is constant for all particles at a certain time or location but varies with time and location, respectively. In the trace-space emittance equation a term of the form

$$
C^{2}\left(\sum x^{2}\right)^{2}\left[\sum \frac{1}{p_{z}^{2}}-\left(\sum \frac{1}{p_{z}}\right)^{2}\right] \neq 0
$$

shows up. Therefore the correlated transverse momentum spread contributes to the trace-space emittance in the presence of a significant energy spread. Before presenting some examples the emittance development in a drift space will be discussed in the next section.

\section{DEVELOPMENT OF THE EMITTANCE IN A DRIFT SPACE}

The position of an individual particle in a drift space develops as

$$
x_{i}=x_{0, i}+x_{i}^{\prime} \cdot z .
$$

For the calculation of the beam emittance the second order moments of the bunch distribution are required, which write as (for simplicity $\bar{x}=\bar{p}_{x}=0$ is assumed)

$$
\begin{gathered}
\frac{1}{n} \sum x^{2}=\frac{1}{n}\left(\sum x_{0}^{2}+2 z \sum x_{0} x^{\prime}+z^{2} \sum x^{\prime 2}\right) \\
\frac{1}{n^{2}}\left(\sum x p_{x}\right)^{2}=\frac{1}{n^{2}}\left(\sum x_{0} p_{x}+z \sum x^{\prime} p_{x}\right)^{2} \\
=\frac{1}{n^{2}}\left[\left(\sum x_{0} p_{x}\right)^{2}+2 z \sum x_{0} p_{x} \sum x^{\prime} p_{x}\right. \\
\left.+z^{2}\left(\sum x^{\prime} p_{x}\right)^{2}\right] .
\end{gathered}
$$

The normalized beam emittance is then given as

$$
\begin{aligned}
\varepsilon_{n, \mathrm{rms}}^{2}=\frac{1}{m_{0}^{2} c^{2} n^{2}}[ & \sum x_{0}^{2} \sum p_{x}^{2}+2 z \sum x_{0} x^{\prime} \sum p_{x}^{2}+z^{2} \sum x^{\prime 2} \sum p_{x}^{2} \\
& \left.-\left(\sum x_{0} p_{x}\right)^{2}-2 z \sum x_{0} p_{x} \sum x^{\prime} p_{x}-z^{2}\left(\sum x^{\prime} p_{x}\right)^{2}\right] .
\end{aligned}
$$

Thus, in order to find a constant emittance in a drift space, the following conditions have to be fulfilled:

$$
\begin{aligned}
& \sum x_{0} x^{\prime} \sum p_{x}^{2}=\sum x_{0} p_{x} \sum x^{\prime} p_{x}, \\
& \sum x^{\prime 2} \sum p_{x}^{2}=\left(\sum x^{\prime} p_{x}\right)^{2} .
\end{aligned}
$$

This is in general only the case if the energy spread in the beam is sufficiently small. Otherwise a correlation between transverse particle position and energy builds up according to Eq. (20). The induced emittance growth is of cause correlated; thus it is in principle reversible, and it does not violate Liouville's theorem because it shows up only in the projected emittance and not in the six dimensional phase space volume. The emittance variation is also negligible in case that the beam size does not change significantly over the distance $z$, which is equivalent to

$$
\begin{aligned}
\sum x_{0} x^{\prime} & \approx \sum x_{0} p_{x} \approx 0, \\
z^{2} \sum x^{\prime 2} & \approx 0, \\
z^{2} \sum x^{\prime} p_{x} & \approx 0 .
\end{aligned}
$$

For an equivalent calculation of the trace-space emittance $p x$ has to be replaced by $x^{\prime}$ in Eq. (23), which 
directly shows that the trace-space emittance is always constant in a drift section. This means that the variation of the correlation term as given by Eq. (19) and the correlated growth of the spot size as described by Eq. (21) cancel.

\section{EXAMPLES}

In the previous section it has been shown that the beam emittance is not constant in a drift section in the presence of a significant energy spread due to the buildup of a correlation of the transverse particle positions and the longitudinal particle momentum, while the trace-space emittance is always constant in a drift section. On the other hand it was found that the emittance and the tracespace emittance are equal if the correlated transverse momentum spread is close to zero. Hence it is clear that the trace-space emittance changes when the transverse momentum spread or the energy spread is changed.
Figure 2 compares the development of the normalized emittance and the normalized trace-space emittance for the case of varying energy spread. A beam with $5 \mathrm{mrad}$ correlated beam divergence and zero energy spread is launched. A cavity at $z=0.2 \mathrm{~m}$ introduces a correlated energy spread of $2 \times 10^{-3}$ which is compensated by another cavity at $z=1.0 \mathrm{~m}$. A third cavity at $z=1.2 \mathrm{~m}$ introduces a correlated energy spread of $-2 \times 10^{-3}$ which is again compensated by a fourth cavity at $z=$ $2.0 \mathrm{~m}$. For this calculation the transverse fields in the cavities are switched off, so that the beam divergence does not change.

As previously discussed a correlated, reversible emittance growth shows up in the presence of a large energy spread and a large beam divergence in the drift sections. On the contrary the trace-space emittance is constant in the drift sections but jumps in the cavities where the energy spread is changed. The height of the jumps scales

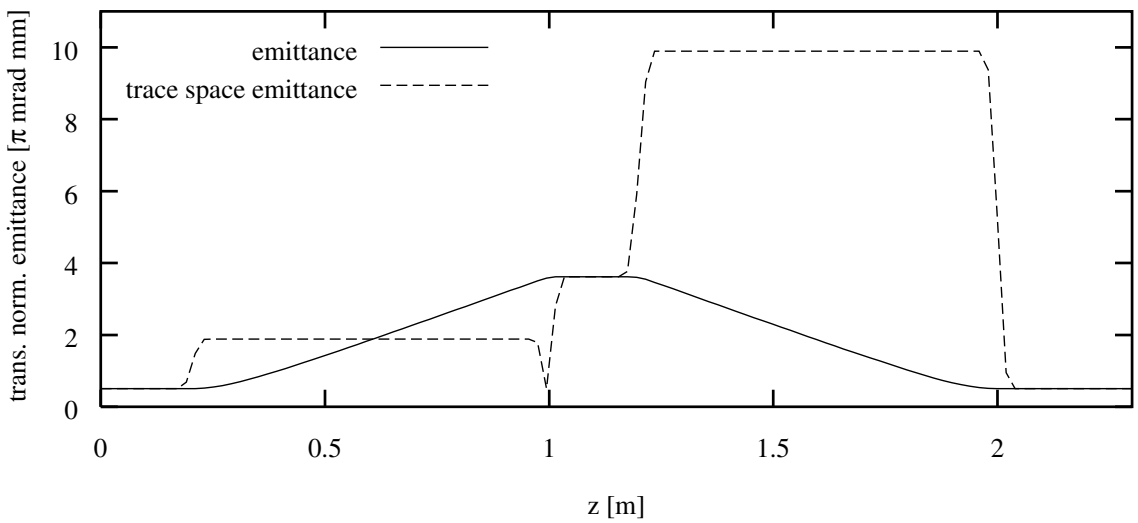

FIG. 2. Comparison of the normalized emittance and the normalized trace-space emittance. A beam with $5 \mathrm{mrad}$ correlated beam divergence is launched. A cavity at $z=0.2 \mathrm{~m}$ introduces a correlated energy spread of $2 \times 10^{-3}$ which is compensated by another cavity at $z=1.0 \mathrm{~m}$. A third cavity at $z=1.2 \mathrm{~m}$ introduces a correlated energy spread of $-2 \times 10^{-3}$ which is again compensated by a fourth cavity at $z=2.0 \mathrm{~m}$. See text for details.

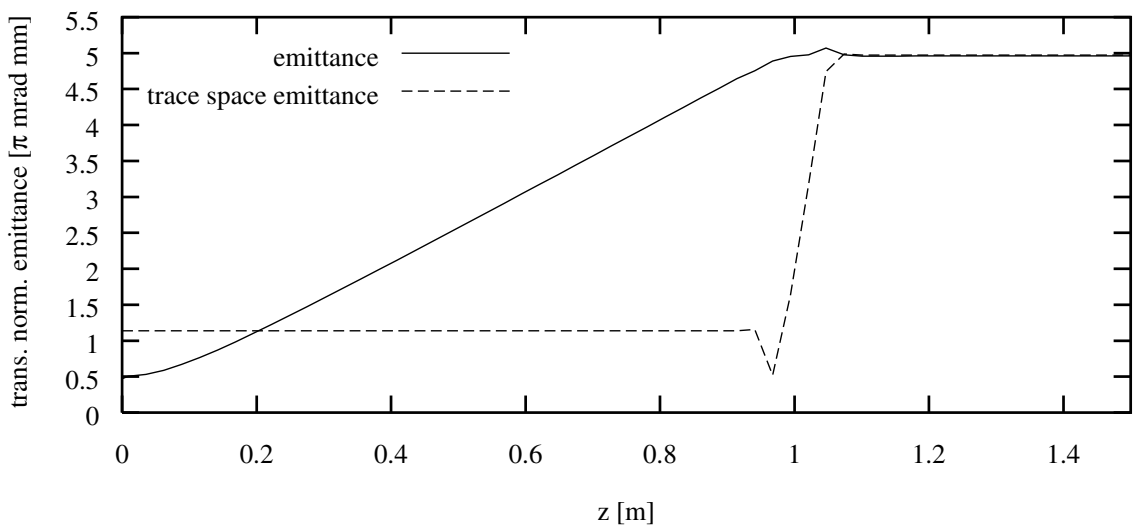

FIG. 3. Comparison of the normalized emittance and the normalized trace-space emittance. A beam with 5 mrad correlated divergence and $2 \times 10^{-3}$ incoherent energy spread is launched. A quadrupole at $z=1 \mathrm{~m}$ compensates the correlated beam divergence. See text for details. 


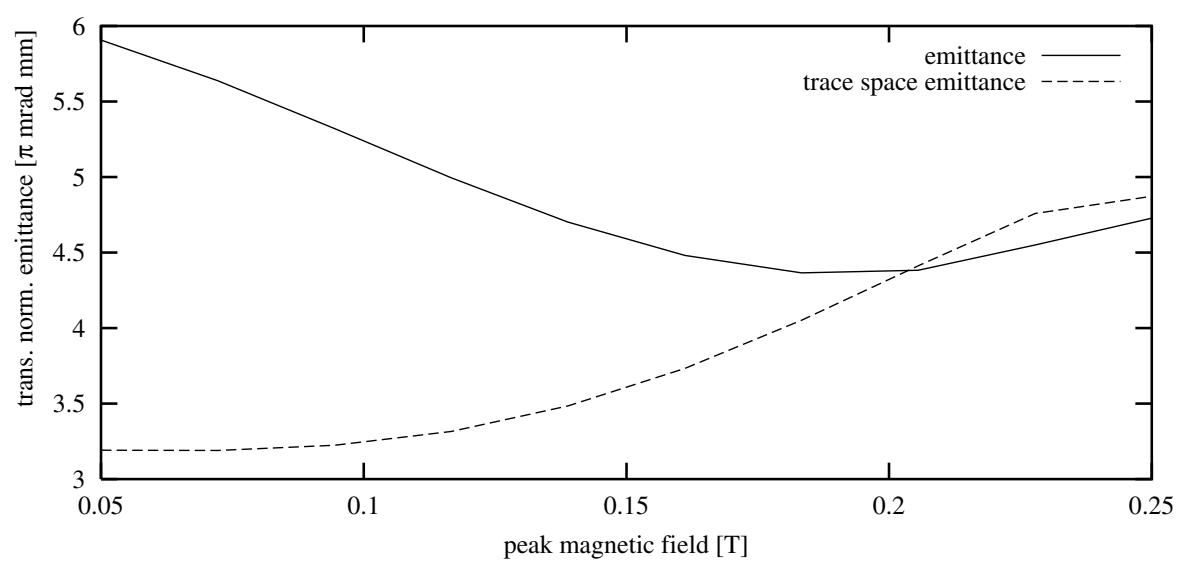

FIG. 4. Comparison of the normalized emittance and the normalized trace-space emittance for the case of a solenoid scan at an rf gun setup.

with the length of the drift in front of the cavity. The trace-space emittance and the emittance are equal when the energy spread is close to zero. In Fig. 3 the effects of a varying correlated beam divergence on the normalized beam emittance and on the normalized trace-space emittance are compared. A beam with $5 \mathrm{mrad}$ correlated divergence and $2 \times 10^{-3}$ incoherent energy spread is launched. A quadrupole at $z=1 \mathrm{~m}$ compensates the correlated beam divergence. In order to compensate the emittance growth in a subsequent drift of equal length the $p x$ of all particles has to be replaced by $-p x$. This cannot be achieved by a single quadrupole since the change of the transverse momentum depends on the longitudinal particle momentum. Similar to the previous example the trace-space emittance and the emittance are equal when the beam divergence is close to zero.

The practical relevance of the described effect is demonstrated in Fig. 4 where the emittance behind an rf gun ( $1 \mathrm{~m}$ downstream of the cathode) as a function of the strength of the focusing solenoid is shown. While the difference of emittance and trace-space emittance is neg- ligible at higher energy and optimized injector parameters, significant differences can appear during parameter scans, which are typically performed during the characterization of an rf gun. For this calculation the rf phase of the gun has been set $10^{\circ}$ off the phase of maximum energy gain, in order to introduce some energy spread.

\section{EFFECT OF THE LONGITUDINAL COORDINATE ON THE TRANSVERSE EMITTANCE}

So far the effect of the longitudinal coordinate on the transverse emittance has been neglected. In accelerators the beam emittance is derived, for example, from spot size measurements with a screen. Thus the transverse coordinates of each particle are recorded when the particle passes a certain longitudinal position, which happens at different times for different particles. In the following this will be called a local emittance. On the contrary it is convenient to calculate the emittance at a certain time, i.e., with different longitudinal positions of

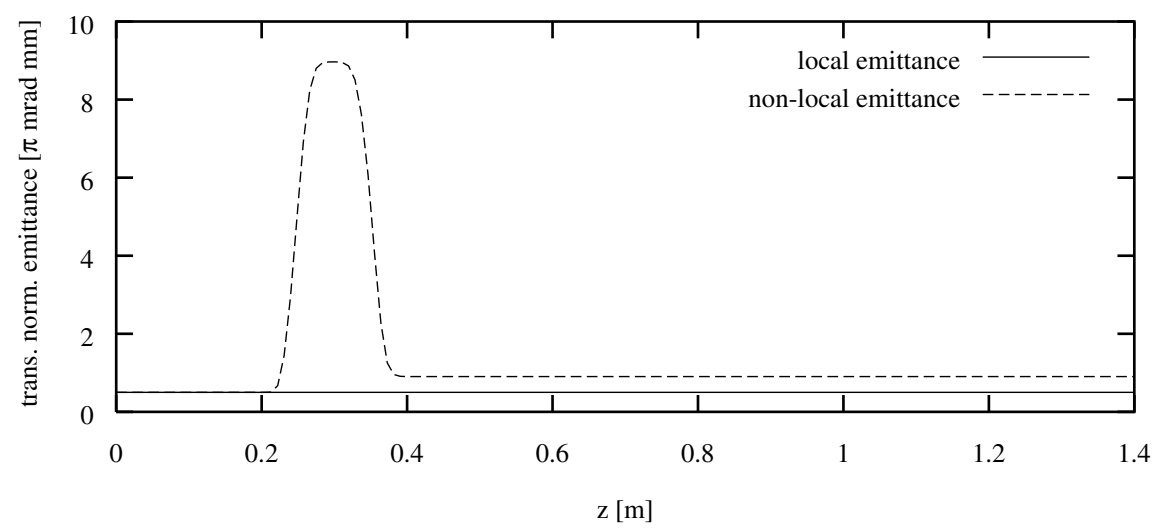

FIG. 5. Development of the local and nonlocal emittance in a beam line with a quadrupole centered at $z=0.3 \mathrm{~m}$. The nonlocal emittance shows a large correlated emittance contribution inside the quadrupole but also some emittance growth in the drift behind the magnet. 
the individual particles, in computer codes which have the time as a free variable. The latter procedure introduces obviously large correlated emittance contributions, for example, when the emittance is calculated inside a beam line element where the head of the bunch has integrated over fields which the tail has not yet seen. Figure 5 compares the development of the local and the nonlocal emittance in a beam line with a quadrupole centered at $z=0.3 \mathrm{~m}$. In the quadrupole a large correlated emittance growth is seen in the nonlocal emittance, but also in the drift behind the quadrupole a small emittance contribution remains, since the head of the divergent (convergent) bunch is slightly larger (smaller) than the tail. This corresponds to a fanlike structure in phase space. The local emittance calculation is obviously advantageous since it avoids confusing correlated emittance contributions, however, the difference of local and nonlocal emittance is in drift sections, where the emittance is normally measured, in many cases negligible small. The examples of the previous section present the development of the local emittance.

\section{CONCLUSION}

In the first paragraph the significance of rms values for the emittance calculation and emittance measurements is emphasized. The discussion of the development of the emittance showed that the emittance is not necessarily constant in a drift space and that large differences between emittance and trace-space emittance may appear. Some beam parameters in the discussed examples are somewhat extreme in order to show clearly the effect of the energy spread and of the beam divergence on the beam emittance. However, similar effects might be expected in bunch compressor sections where the energy spread can be large. In gun sections of high brightness beam applications the effect is small in case of optimized beam parameters, but significant differences between (measured) trace-space emittance and (calculated) emittance can appear during parameter scans. Sources with large divergence and energy spread, like positron sources and plasma electron sources, are obviously sensitive to the described effect. The difference of emittance and trace-space emittance has two aspects. First it is obvious that the beam emittance, rather than the trace-space emittance, should be calculated in tracking simulations in order to get meaningful results. The trace-space emittance shows an unphysical behavior and is no adequate tool to optimize the beam line settings. Second it is important to check whether the beam emittance or the trace-space emittance is measured and, in the latter case, to make sure that the trace-space emittance and the beam emittance are equal, i.e., that the energy spread is sufficiently small.

\section{ACKNOWLEDGMENTS}

This work has been stimulated by discussions with numerous colleagues to whom I am indebted. Especially
I would like to mention Georg Hoffstaetter and Zhenghong Li.

\section{APPENDIX}

\section{Derivation of the rms emittance formula}

In order to remove the linearly correlated transverse momentum from a distribution of particles in the transverse phase space a straight line fit $p_{x}=m \cdot x$ is made to the distribution which yields the inclination $m$ of the distribution. The correlation is removed from the distribution by setting

$$
\tilde{p}_{x, i}=p_{x, i}-m \cdot x_{i}, \quad m=\frac{\left\langle x p_{x}\right\rangle}{\left\langle x^{2}\right\rangle} .
$$

In the new variables the emittance is just given by the product of the rms values of both coordinates as

$$
\varepsilon_{n, \mathrm{rms}}=\frac{1}{m_{0} c} \sqrt{\left\langle x^{2}\right\rangle\left\langle\tilde{p}_{x}^{2}\right\rangle} .
$$

Introducing Eq. (A1) into Eq. (A2) gives the rms emittance formula Eq. (11). Note that Eq. (A1) defines a shearing in phase space and that the line defined by $m$ corresponds to the angle $\varphi_{\text {cor }}$ of Eq. (8).

\section{Relation of statistical beam parameters to the optical functions}

The Courant Snyder invariant is written as

$$
\varepsilon_{\mathrm{rms}}=\gamma x^{2}+2 \alpha x x^{\prime}+\beta x^{\prime 2},
$$

where $\alpha, \beta$, and $\gamma$ are the twiss parameters.

With $\beta \gamma-\alpha^{2}=1$ one can write

$$
x_{1,2}^{\prime}=-\frac{\alpha}{\beta} x \pm \sqrt{\frac{\varepsilon_{\mathrm{rms}}}{\beta}-\frac{x^{2}}{\beta^{2}}} .
$$

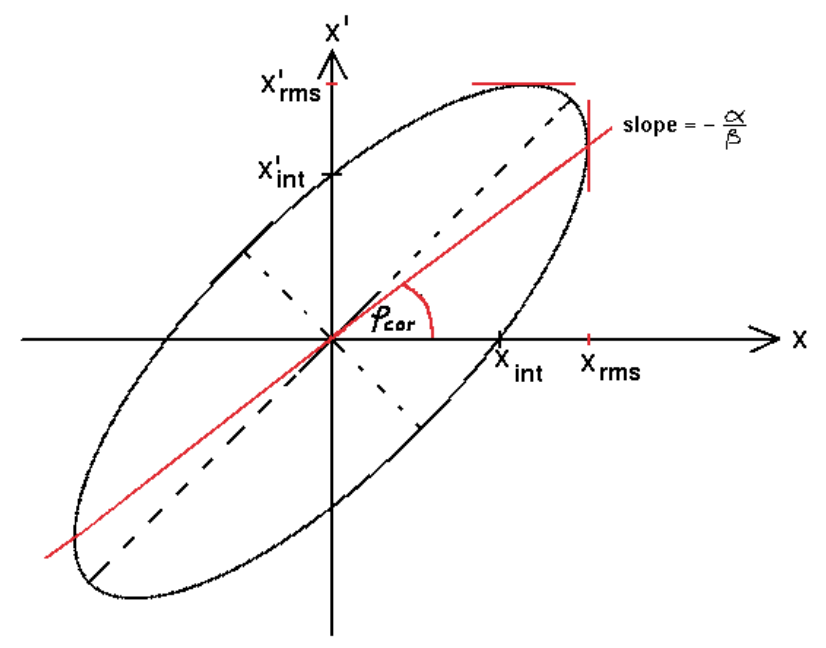

FIG. 6. (Color) Phase space ellipse with important points on the circumference of the ellipse. 
The correlated beam divergence of the ellipse is given by $-\alpha / \beta=m=\left\langle x p_{x}\right\rangle /\left\langle x^{2}\right\rangle$. If $-\alpha / \beta$ is negative the beam is convergent otherwise it is divergent. Figure 6 shows a phase space ellipse and indicates points on the circumference of the ellipse.

The indicated points are related to the twiss parameters and to statistical beam parameters by

$$
\begin{aligned}
& x_{\mathrm{rms}}=\sqrt{\varepsilon_{\mathrm{rms}} \cdot \beta}, \quad x_{\mathrm{rms}}^{\prime}=\sqrt{\varepsilon_{\mathrm{rms}} \cdot \gamma}, \\
& x_{\mathrm{int}}=\sqrt{\frac{\varepsilon_{\mathrm{rms}}}{\gamma},} \quad x_{\mathrm{int}}^{\prime}=\sqrt{\frac{\varepsilon_{\mathrm{rms}}}{\beta}} .
\end{aligned}
$$

